\title{
Ultrahigh numerical aperture meta-fibre for flexible optical trapping
}

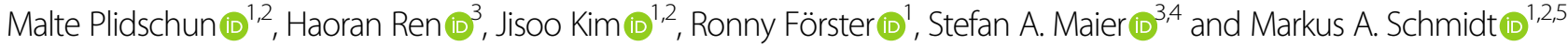

\begin{abstract}
Strong focusing on diffraction-limited spots is essential for many photonic applications and is particularly relevant for optical trapping; however, all currently used approaches fail to simultaneously provide flexible transportation of light, straightforward implementation, compatibility with waveguide circuitry, and strong focusing. Here, we demonstrate the design and 3D nanoprinting of an ultrahigh numerical aperture meta-fibre for highly flexible optical trapping. Taking into account the peculiarities of the fibre environment, we implemented an ultrathin meta-lens on the facet of a modified single-mode optical fibre via direct laser writing, leading to a diffraction-limited focal spot with a recordhigh numerical aperture of up to $N A \approx 0.9$. The unique capabilities of this flexible, cost-effective, bio- and fibre-circuitrycompatible meta-fibre device were demonstrated by optically trapping microbeads and bacteria for the first time with only one single-mode fibre in combination with diffractive optics. Our study highlights the relevance of the unexplored but exciting field of meta-fibre optics to a multitude of fields, such as bioanalytics, quantum technology and life sciences.
\end{abstract}

\section{Introduction}

Strong focusing of light onto diffraction-limited spots is one of the fundamental prerequisites for a vast number of optical applications, including high-resolution imaging, microscopy, optical manipulation, and material processing through optical lithography. In particular, precise optical control and manipulation of micro- and nanoscopic objects by focused laser beams ${ }^{1}$ have led to sophisticated applications in biophotonics ${ }^{2-5}$, microfluidics $^{6-8}$, and quantum technology ${ }^{9,10}$. Here, stable and robust optical trapping demands a strong gradient force along the three spatial directions to provide a strong trapping force in the radial direction and to counterbalance the scattering force along the axial direction. As such, tight light focusing is conventionally achieved by using microscope objectives with a very high numerical aperture $(\mathrm{NA}>0.8)$; however, these optical components

\footnotetext{
Correspondence: Markus A. Schmidt (markus.schmidt@leibniz-ipht.de) ${ }^{1}$ Leibniz Institute of Photonic Technology, 07745 Jena, Germany

${ }^{2}$ Abbe Center of Photonics and Faculty of Physics, FSU Jena, 07745 Jena, Germany

Full list of author information is available at the end of the article
}

are bulky and suffer from a lack of flexibility and integrability, poor remote operating capabilities, and high cost and demand for large-volume infrastructure.

Optical fibres open up the possibility to address the abovementioned limitations and represent a flexible, remotely operable, and low-cost photonic platform. In particular, the use of single-mode fibres (SMFs) offers a major advantage for producing output beams with welldefined optical properties. Unlike multimode fibres (MMFs) that include many modes, SMFs support only the low-loss fundamental mode. This gives rise to a wellcontrolled output beam that is insensitive to external influences and yields a uniform phase profile, which is essential for wavefront manipulation. Moreover, SMFs allow remote distances to be reached when light propagation is unaffected by the length of the optical fibre, while MMFs are highly susceptible to fibre bending. Nevertheless, the use of optical fibres in focusing applications and particular optical trapping is fundamentally limited by the divergence of light emerging from the fibre facet. With respect to optical trapping, several approaches have been proposed to overcome divergence, including

\section{(c) The Author(s) 2021}

(c) (i) Open Access This article is licensed under a Creative Commons Attribution 4.0 International License, which permits use, sharing, adaptation, distribution and reproduction cc) in any medium or format, as long as you give appropriate credit to the original author(s) and the source, provide a link to the Creative Commons license, and indicate if changes were made. The images or other third party material in this article are included in the article's Creative Commons license, unless indicated otherwise in a credit line to the material. If material is not included in the article's Creative Commons license and your intended use is not permitted by statutory regulation or exceeds the permitted use, you will need to obtain permission directly from the copyright holder. To view a copy of this license, visit http://creativecommons.org/licenses/by/4.0/. 
dual-fibre arrangements with counterpropagating beams $^{4,11-13}$ and MMFs in combination with an iterative adaptive compensation technique ${ }^{14}$. Although trapping has been successfully demonstrated, the associated systems include complicated and cumbersome optical components that are not suitable for in vivo applications.

Recent advances in nanotechnology provide unprecedented opportunities to shape optical beams via the use of ultrathin meta-surfaces that are composed of subwavelength elements, and, thus, allow digitization and tailoring of wavefronts at interfaces ${ }^{15,16}$. In this context, implementation of meta-lenses on fibre tips for tight light focusing has been realized via, e.g., focused ion-beam milling ${ }^{17,18}$ and chemical etching ${ }^{19}$; however, the realized meta-lenses only reach a maximum NA of 0.37 (ref. ${ }^{18}$ ). On the other hand, three-dimensional (3D) direct laser writing (DLW) based on two-photon polymerization, offers an ideal platform to optically digitalize arbitrary 3D nanostructures on substrates that are difficult to handle otherwise ${ }^{20,21}$. Although first attempts have recently been made to optically digitize diffractive Fresnel lenses on a fibre tip ${ }^{22}$, a dual fibre arrangement for optical trapping is still required due to the limited maximum NA of 0.7. Therefore, implementing an ultrahigh NA meta (UNM)lens on a fibre tip that allows for tight focusing of light in an in vivo environment remains an open challenge, while such an arrangement represents a powerful approach for a multitude of applications, examples of which include flexible optical trapping and high-resolution scanning microscopy.

Here, we demonstrate the design and 3D nanoprinting of an UNM on the facet of a functionalized SMF, underpinning an UNM-fibre device for flexible optical trapping based on a single single-mode fibre (Fig. 1a).
Specifically, we show the design and experimental realization of UNMs with spatial resolutions of down to $300 \mathrm{~nm}$, taking into account the peculiarities of the fibre environment. By reaching record-high numerical apertures of up to $\approx 0.9$ and diffraction-limited spots (Fig. 1b), we reveal the capabilities of our device by optically trapping microbeads and biologically relevant species for the first time with a single SMF in combination with diffractive optics. The implementation relies on 3D-DLW in combination with a functionalized SMF including a submillimetre piece of MMF for beam expansion to accommodate the full numerical aperture of the UNM (Fig. 2a). This allows us to expand the cross-section of the output beam of the SMF to a significantly larger lateral dimension $(\sim 95 \mu \mathrm{m}$ in diameter), which would otherwise be too small $(\sim 4 \mu \mathrm{m}$ in diameter) to enable sufficient wavefront manipulation. Flexible remote optical trapping of silica microbeads (Fig. 1c) and Escherichia coli (E. coli) bacteria (Fig. 1d) is experimentally demonstrated, confirming the unique focusing performance of our device (see Movies S1-S6).

\section{Results \\ Design of an UNM-enhanced meta-fibre}

Interfacing an UNM with an optical fibre requires careful geometric and phase design to include the specific circumstances of the fibre environment in terms of both beam properties and geometric constraints.

\section{Meta-lens design}

Experimental implementation of the UNM used here with a focal point at an axial distance $z=f$ relies on 3D-DLW, requiring discretization of a hyperbolic phase
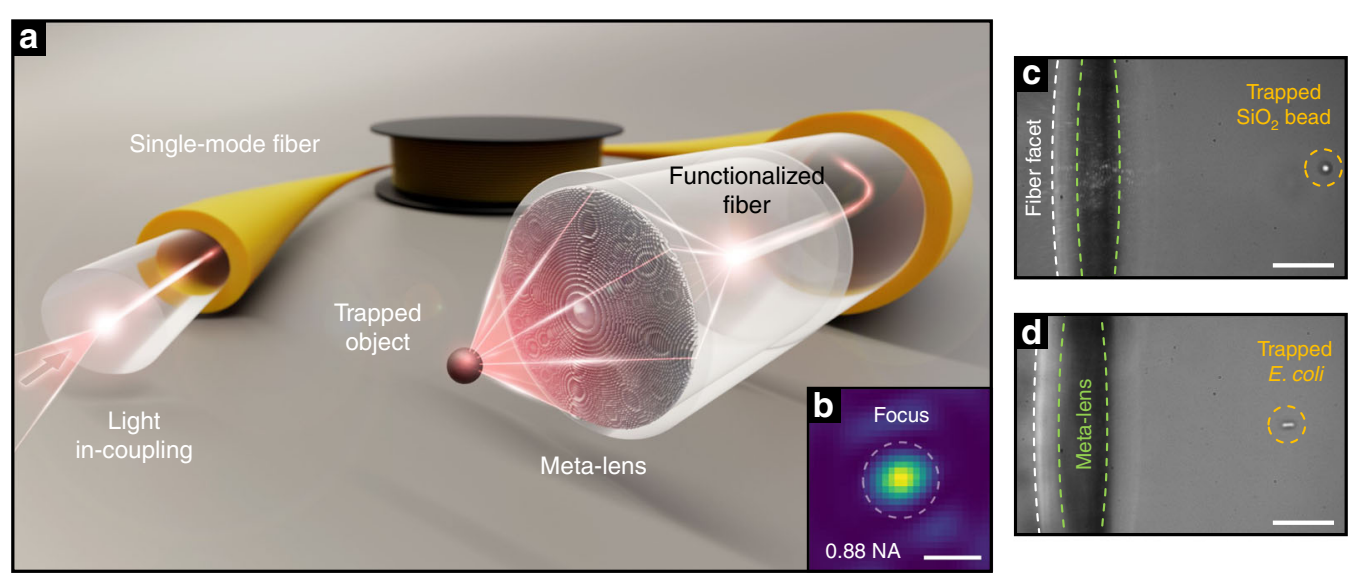

Fig. 1 Principle of an ultra-high NA meta-fibre for flexible optical trapping. a Illustration of the device including a 3D nanoprinted meta-lens located on the tip of an optical fibre to trap particles. b Example of a measured radial focal plane in water (scale bar: $500 \mathrm{~nm}$, wavelength of $660 \mathrm{~nm}$, the dashed line indicates the width of the fitted Airy function). c Micrograph of a $2 \mu \mathrm{m}$ silica bead and (d) an Escherichia coli (E. coli) bacterium optically trapped with a single fibre UNM in water imaged from the side (scale bars $15 \mu \mathrm{m}$ ) 


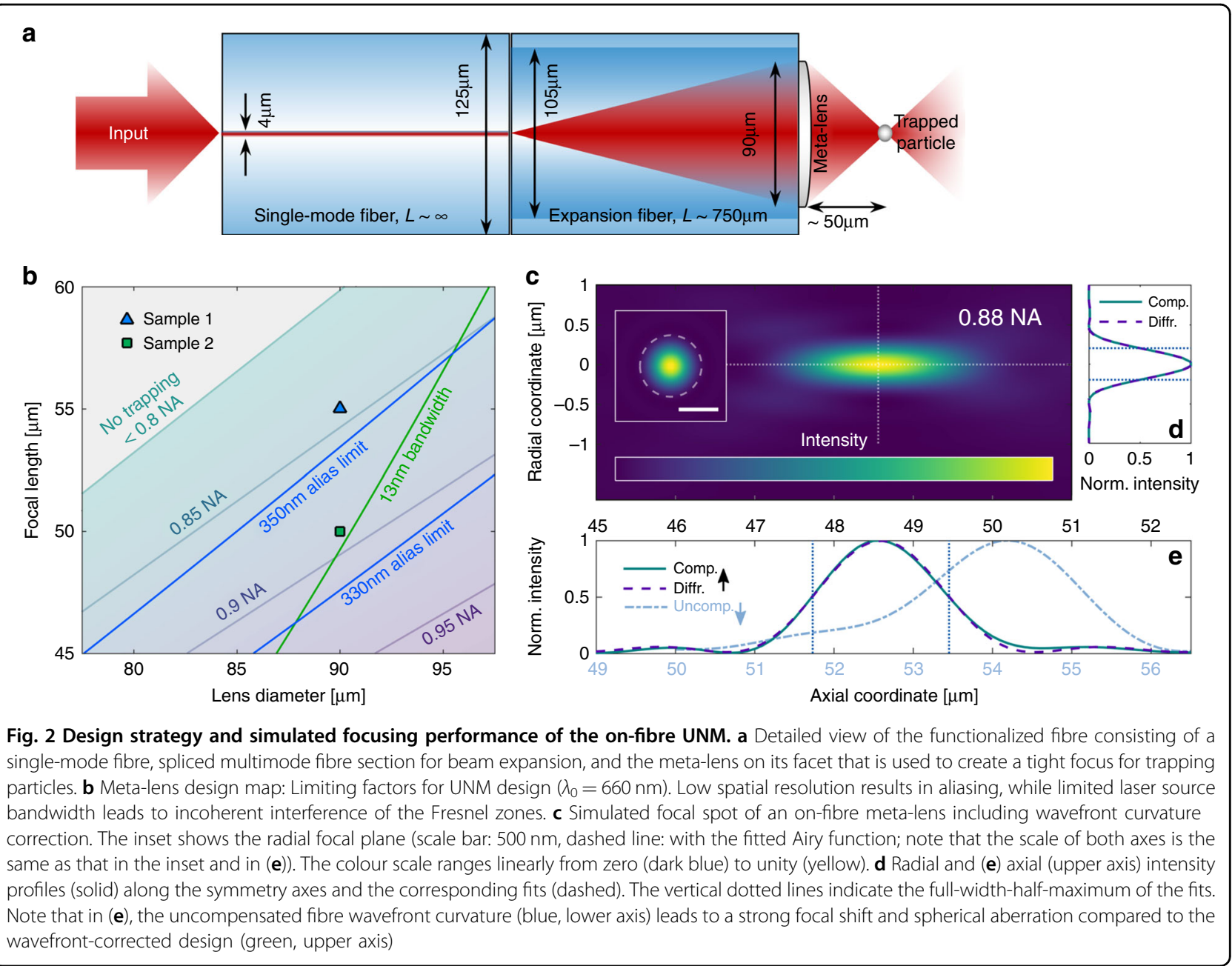

$\phi_{\text {hyp }}(r, f)=-2 \pi n / \lambda_{0} \cdot\left(\sqrt{r^{2}+f^{2}}-f\right) \quad(n: \quad$ refractive index of the medium, $\lambda_{0}$ : vacuum wavelength, $r$ : radial coordinate). The phase profile $\phi$ is discretized in steps of $2 \pi$ into Fresnel zones via $\phi_{\text {kin }}=\bmod (\phi, 2 \pi)$, leading to a kinoform-type phase distribution ${ }^{23-25}$ (details in Supplementary Results 1). Note that the hyperbolic phase profile exhibits less critical curvature towards the edges than its parabolic counterpart, and is, thus, used throughout this work. In contrast to a multilevel diffractive Fresnel lens ${ }^{23,24}$ with locally adaptive sampling, the globally constant in-plane discretization $\Delta x$ establishes limits to the interpixel phase change that can be spatially resolved $\left(\left|\phi^{\prime}\right| \approx|\Delta \phi / \Delta x|<\pi / \Delta x\right)$, which is defined by the Nyquist-Shannon theorem ${ }^{26}$, and reduces the effectively usable lens diameter fraction and connected NA for the case of undersampling, i.e., aliasing. Here, we choose a globally constant spatial resolution of $\Delta x=300 \mathrm{~nm}$ to ensure alias-free operation at $\lambda_{0}=660 \mathrm{~nm}$ (see Supplementary Results 2 and Fig. S1 for details), which results in a constant in-plane pitch for the elements (Fig. S6), similar to the arrangement of a metasurface. This is different from a multilevel diffractive Fresnel lens ${ }^{23,24}$, which relies on locally variable in-plane pitches that yield a limited NA when the spatial resolution of the implementation approach (in the present case of 3D nanoprinting technology) is on the order of hundreds of nanometres. To efficiently make use of the entire lens cross-section (i.e., NA, chosen here to be $2 r=90 \mu \mathrm{m}$ ), constructive interference of wavefronts emerging from all lens elements is required, which is limited by the coherence length of the laser source $L_{\mathrm{coh}}=\lambda_{0}{ }^{2} /\left(n \Delta \lambda_{0}\right)$ (bandwidth $\left.\Delta \lambda_{0}\right)$. Note that the highest lens performance in terms of NA is achieved for the case when all Fresnel rings are taken into account, i.e., if $L_{\text {coh }}>\lambda_{0} N_{\text {Fres }}$ ( $N_{\text {Fres }}$ : number of Fresnel rings, see details in Supplementary Results 3). All mentioned issues allow us to define a meta-lens design map (Fig. 2b) including the alias limit and bandwidth of the laser source used $\left(\Delta \lambda_{0}=13 \mathrm{~nm}\right)$, showing that the two experimentally targeted 
configurations (sample 1: $\Delta x_{1}=330 \mathrm{~nm}, f_{1}=55 \mu \mathrm{m}$; sample 2: $\left.\Delta x_{2}=300 \mathrm{~nm}, f_{2}=50 \mu \mathrm{m}\right)$ are in line with the limits imposed by the nanoprinting process.

\section{Fibre-wavefront compensation}

Particular emphasis must be placed on the divergence of the beam emitted by the fibre to achieve maximum meta-lens performance. For conventional step-index fibres, wavefronts are curved with radius $R(z)=z \cdot(1+$ $\left.z_{\mathrm{R}}{ }^{2} / z^{2}\right) \approx z$ for propagation distances significantly larger than the Rayleigh length $\left(z \gg z_{\mathrm{R}} \sim 10 \ldots 30 \mu \mathrm{m}\right.$ in the visible spectrum). Hence, the fibre acts as a negative defocusing lens with $f=-R(z)$ and exhibits its own spherical phase $\phi_{\text {fib }}(r, z)=k \cdot\left(\sqrt{r^{2}+z^{2}}-z\right)$, leading to an additional contribution to the overall phase $\phi_{\text {tot }}(r, f$, $z)=\phi_{\text {hyp }}(r, f)+\phi_{\text {fib }}(r, z)$. It is straightforward to compensate for this spherical aberration (i.e., phase anomalies) by using DLW (see Fig. S2 for details), thus representing a key feature.

Figure 2c shows the simulated beam profile for a hyperbolic-phase meta-lens (sample 2, NA=0.88), including the mentioned wavefront correction $\phi_{\text {tot }}(r, f, L)-$ $\phi_{\mathrm{fib}}\left(r, L_{\mathrm{min}}=700 \mu \mathrm{m}\right)$. Note that the output mode from the fibre freely expands in the MMF section across a length $L \sim 750 \mu \mathrm{m}$ to fill the entire lens aperture. The use of an MMF is essential, as reflections and aberrations at the fibre junction are significantly reduced due to the matched refractive indices of both fibres, which is different from the case when a pure silica rod is used for beam expansion. Due to experimental tolerances in fibre cutting, a minimum length $L_{\min }=700 \mu \mathrm{m}$ for the expansion fibre is assumed in the meta-lens design, leading to an experimentally overcompensated fibre aberration, which, in general, has a less severe impact on lens performance than under-compensation. This is because a short section introduces stronger wavefront curvature than that possible for an overestimated design. Furthermore, the overestimation accounts for possible deviations of the actual wavefront from the theoretical fibre wavefront assumed in the design for the correct length. According to diffraction theory, the intensity profiles along the symmetry lines (Fig. 2d, e) were fitted along the radial $I(r) \sim$ jinc $^{2}\left(2 \pi r / \lambda_{0}\right.$.NA $)$ and axial $I(z) \sim \operatorname{sinc}^{2}\left((z-f) / \lambda_{0} \cdot\left(n-\sqrt{n^{2}-\mathrm{NA}^{2}}\right)\right)$ directions to retrieve the numerical aperture ${ }^{27}$ (see details in Supplementary Results 4). Note that an uncorrected phase profile reduces the NA by $\sim 0.1$ to $<0.8$, including a shift of $\Delta f \sim 5 \mu \mathrm{m}$ in the axial focal position. Overall, the results reveal that the specific design procedure outlined here allows for the implementation of metalens-enhanced fibre devices with unprecedented high numerical apertures of up to $\mathrm{NA} \approx 0.9$.

\section{Meta-fibre fabrication and characterization Implementation}

A $50 \mathrm{~cm}$-long piece of conventional SMF (Thorlabs $630 \mathrm{HP}$, mode diameter $4 \mu \mathrm{m}$, single-mode at $\lambda_{0}=$ $660 \mathrm{~nm}$ ) was fusion spliced to an MMF (Thorlabs FG105LVA, core diameter $105 \mu \mathrm{m}$ ) and used for beam expansion to exploit the full cross-section of the metalens (Fig. 3a). The spliced MMF is then cleaved to a length of approximately $700 \mu \mathrm{m}$ (sample 1: $L_{1} \sim 720 \mu \mathrm{m}$, sample 2: $\left.L_{2} \sim 750 \mu \mathrm{m}\right)$, resulting in a beam $(\mathrm{NA} \approx 0.09)$ spanning across the actual meta-lens aperture $(2 r=90 \mu \mathrm{m}$, images of the fundamental mode output are shown in Fig. S5). UNM implementation includes a silanization step to improve adhesion (see details in "Materials and methods") and 3D nanoprinting (Nanoscribe $\mathrm{GmbH}$ ) using an IPDip photoresist. All implemented lenses have diameters of $2 r=90 \mu \mathrm{m}$, while two types of samples were produced (sample 1: $\Delta x_{1}=330 \mathrm{~nm}, f_{1}=55 \mu \mathrm{m}(\mathrm{NA}=0.84)$; sample 2: $\Delta x_{2}=300 \mathrm{~nm}, f_{2}=50 \mu \mathrm{m}(\mathrm{NA}=0.88)$, Fig. $\left.3 \mathrm{~b}, \mathrm{c}\right)$. The final height of a meta-lens is $h \sim 3 \mu \mathrm{m}$, requiring an approximate printing time of $1 \mathrm{~h}$ (see Fig. S6 for close-up micrographs of the structure of a 3D nanoprinted UNM). The measured transmission through a UNM is $>50 \%$ in the visible spectrum and significantly increases for longer wavelengths due to reduced scattering losses.

\section{Performance of on-fibre UNM}

The focusing performance of the meta-lens-enhanced fibre was determined by coupling light from a laser diode (Thorlabs L660P120, $\lambda_{0}=660 \mathrm{~nm}$, bandwidth $\Delta \lambda_{0}=$ $13 \mathrm{~nm}$ ) into the sample and immersing its tip into the water while measuring the transverse beam profile at different distances from the UNM surface (step size $\Delta z=$ $100 \mathrm{~nm}$ ) with a high-NA water-dipping objective (Nikon $60 \times, \mathrm{NA}=1.0$, see "Materials and methods" for further details). At each position, three micrographs with different exposure times were taken and combined to create a high-dynamic-range (HDR) image with a low noise floor of $<10^{-5}$.

As an example, Fig. 3d-g shows the measured beam profile of sample 2, revealing extremely tight and diffraction-limited focusing (Fig. 3d) with the remarkable agreement with the beam profile of the designed UNM (Fig. 2c). The individual focal planes (see Fig. S7) are in excellent agreement with the azimuthal average presented in Fig. 3e and show no distortions into either of the directions. For a qualitative performance comparison, the measured intensity distributions along the symmetry axes of the focus (Fig. 3f: radial, Fig. 3g: axial) are fitted (dashed lines) using the same procedure applied for the meta-lens design, with the vertical dotted lines indicating the FWHM. The resulting values for the numerical aperture are extraordinarily high $(\mathrm{NA} \approx 0.9)$ and match those obtained from simulations (Fig. 2c-e, see Supplementary Results 5 for a 
a

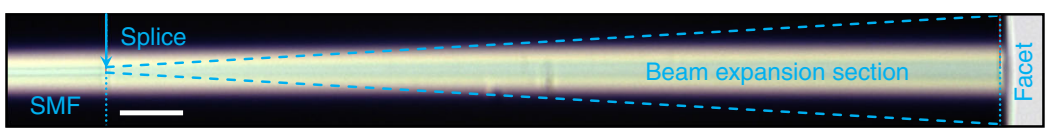

b
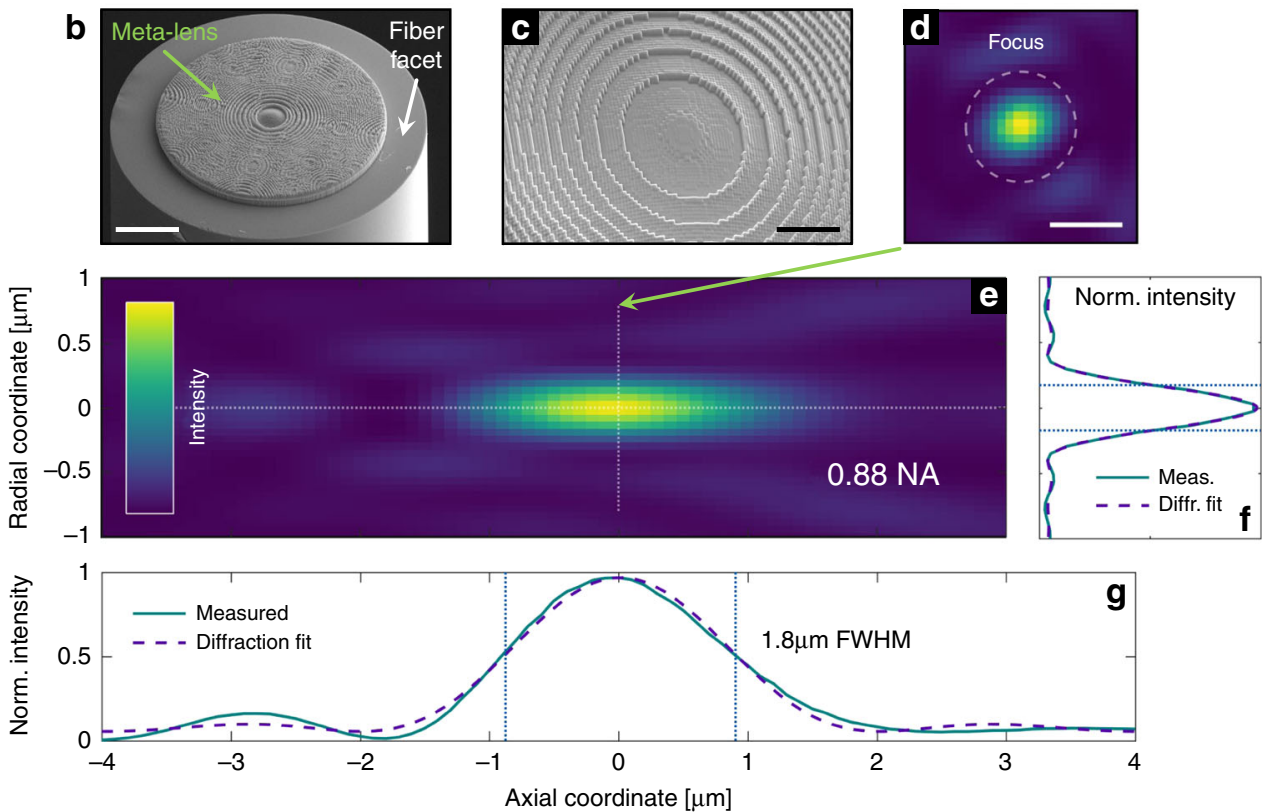

Fig. 3 Measured focusing performance of the implemented UNM-enhanced meta-fibre (sample $2, \lambda_{0}=660 \mathrm{~nm}$ in water). a Microscope image of a functionalized meta-fibre consisting of a single-mode fibre and spliced beam expansion section (scale bar $50 \mu \mathrm{m}$ ). b, c Scanning electron micrographs of an example implemented device (scale bars at $25 \mu \mathrm{m}$ and $10 \mu \mathrm{m}$, respectively). $\mathbf{d}$ Measured cross-section of the beam taken at the focal plane ( $z=0$, scale bar: $500 \mathrm{~nm}$ ). The dashed circle describes the width of a fitted Airy function. e Axial stack for measured beam cross-sections in cylindrical coordinates obtained by transforming each $2 \mathrm{D}$ image into radial symmetry via azimuthal averaging (note that the scale of both axes is the same as that in (d) and $(\mathbf{g})$ ). $\mathbf{f}$ Radial and ( $\mathbf{g}$ ) axial intensity profiles (solid) along the symmetry axes of the focus, with the dashed lines showing the corresponding fits. In all contour plots, the colour scale ranges linearly from zero (dark blue) to unity (yellow)

detailed analysis). Note that even a small spherical aberration at $z= \pm 3 \mu \mathrm{m}$ resulting from overcompensation of the wavefront curvature, i.e., due to the use of $L \sim 750 \mu \mathrm{m}$ instead of $700 \mu \mathrm{m}$, is visible. Specifically, different slopes of the intensity decay before and after the focus are associated with positive spherical aberration and represent a remarkable feature in terms of the agreement between experiment and design.

\section{Meta-fibre-based flexible optical trapping}

The unique properties of the meta-lens functionalized fibre device are demonstrated here by optically trapping freely diffusing micro-objects in water requiring at least NA $>0.8$ (ref. ${ }^{28}$ ). The trapping setup consists of a strong laser diode (Thorlabs L660P120, $\lambda_{0}=660 \mathrm{~nm}$, coupled power at the sample output $P_{\text {out }}=37 \mathrm{~mW}$, Fig. 4a), a windowed liquid chamber containing $2 \mu \mathrm{m}$ silica beads dispersed in water (Fig. 4b) and the functionalized fibre sample (see details in "Materials and methods"). The motion of a trapped object is recorded from the side via a home-built microscope (sketch in Fig. 4c) with a fast frame rate (frequency $1 \mathrm{kHz}$ ) and very low exposure time
$(10 \mu \mathrm{s})$. The trajectory of a trapped bead (Fig. 4d) is determined from its position in each frame via a sophisticated data processing technique (see ref. ${ }^{13}$ for details), yielding the dynamic displacement perpendicular $\left(x_{\perp}(t)\right)$ and parallel $\left(x_{\|}=z\right)$ to the fibre axis.

As for example shown for sample $2(\mathrm{NA}=0.88)$, dielectric microbeads were successfully trapped over desirable timescales (here $>1 \mathrm{~min}$, Fig. $4 \mathrm{~d}$ ) along with all three spatial directions within a region smaller than the area of the focus (Fig. 4e), demonstrating the capabilities of the UNM-enhanced fibre concept with respect to optical trapping (example of a trapped bead is shown in Fig. 4b). It should be mentioned that stable optical trapping was successfully achieved with all fabricated samples over the desired times, while the samples were continuously reused over the course of weeks with no sign of sample degradation observed, making the nanoprinted UNM approach a highly reproducible and reliable concept.

To demonstrate the capabilities of the meta-lensenhanced fibre concept with respect to life science applications, a biologically relevant object (E. coli bacterium) was successfully trapped over the timescale of 


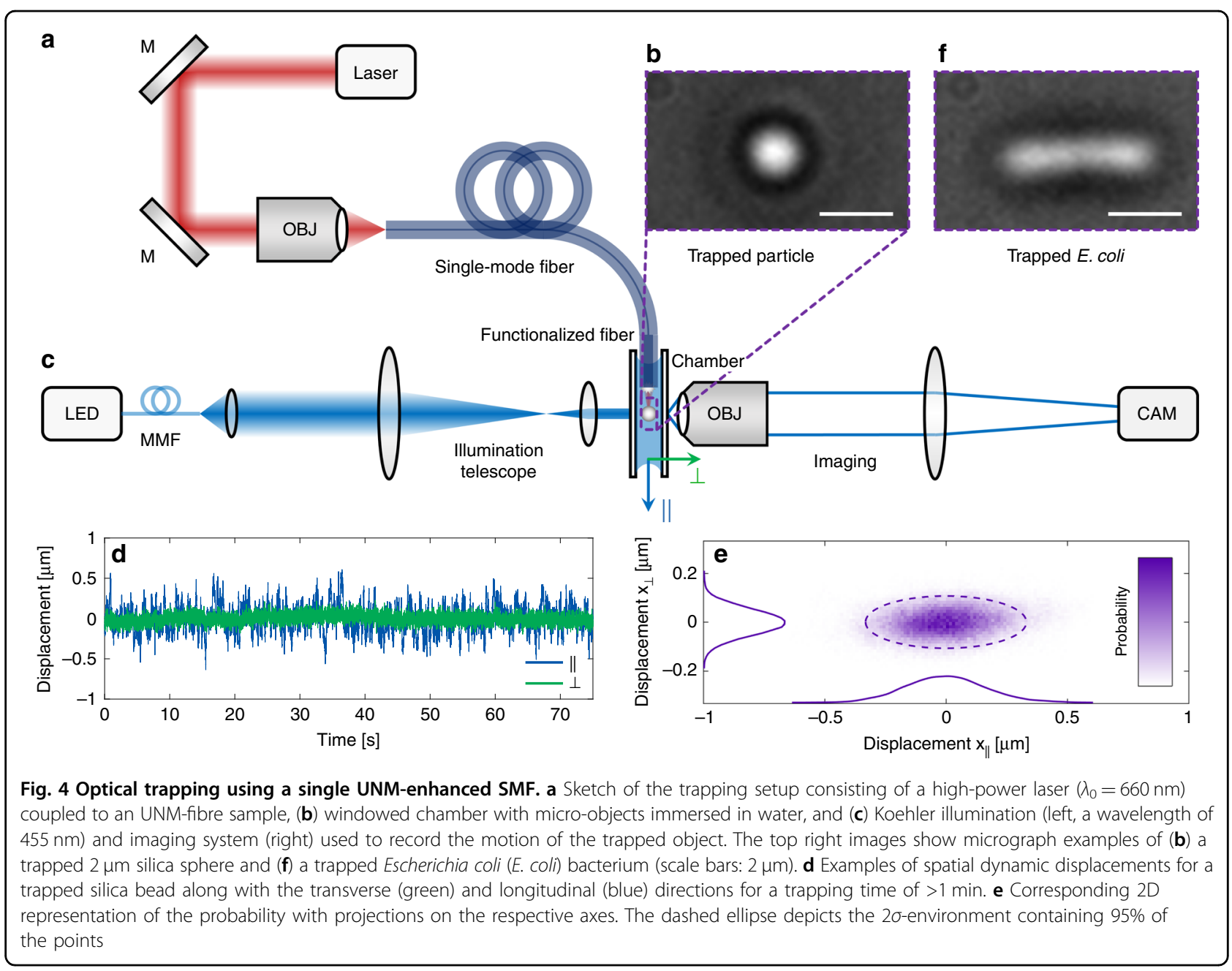

minutes and desirably released from the trap (Figs. $1 \mathrm{~d}$ and 4f, also see Movies S5 and S6). No degradation of either bacterium or meta-lens was observed within subsequent trapping experiments for the same bacterium at powers of up to $37 \mathrm{~mW}$ in the focus. Note that the halo around the trapped objects results from the chosen low NA $(0.16)$ of the Koehler illumination (details in "Materials and methods"). This ensures a great depth of field while maintaining sufficiently high contrast, facilitating image tracking (for details, see ref. ${ }^{13}$ ) owing to larger coverage of the pixels.

To quantitatively assess the trapping performance of the meta-lens-enhanced fibre, we determined the trap stiffness at different power levels by analysing the dynamics of trapped beads. Specifically, power spectral density (PSD) evaluation $^{23}$ and mean-square-displacement (MSD) analysis $^{29}$ (both described in "Materials and methods") were employed for determining the details for the spectral and temporal bead motion characteristics as well as for filtering out potential errors. A key benchmark performance parameter that allows for comparison to other trapping approaches is the optical trap stiffness $\kappa=2 \pi k_{\mathrm{B}} T f_{\mathrm{c}} / D$, which characterizes the strength of the trap potential. This quantity is proportional to the ratio of the two key fit parameters, namely, corner frequency $f_{\mathrm{c}} \sim 1 / \tau_{\mathrm{c}}$ ( $\tau_{\mathrm{c}}$ time constant for confinement inside the trap) and free particle diffusion $D\left(k_{\mathrm{B}} T\right.$ : mean free thermal energy).

The PSD for a measured trajectory (Fig. 4d) is presented in Fig. $5 \mathrm{a}$ and reveals two essential features: the plateau on the low-frequency side results from confinement inside the optical trap, while the linear slope at high frequencies is associated with free particle diffusion. Little to no aliasing from high-frequency noise due to motion blur is visible, resulting from a low exposure time of only $10 \mu \mathrm{s}$. Furthermore, no superimposed secondary oscillatory motion of the trapped bead appearing as a peak in the spectrum is present. In comparison, Fig. $5 \mathrm{~b}$ illustrates the corresponding MSD/lag time dependence, which also shows a plateau for long lag times and a linear slope for short lag times, again describing confinement and free diffusion, respectively. Similar to the PSD analysis, no particle drift on time scales of $>1 \mathrm{~min}$ is visible. 

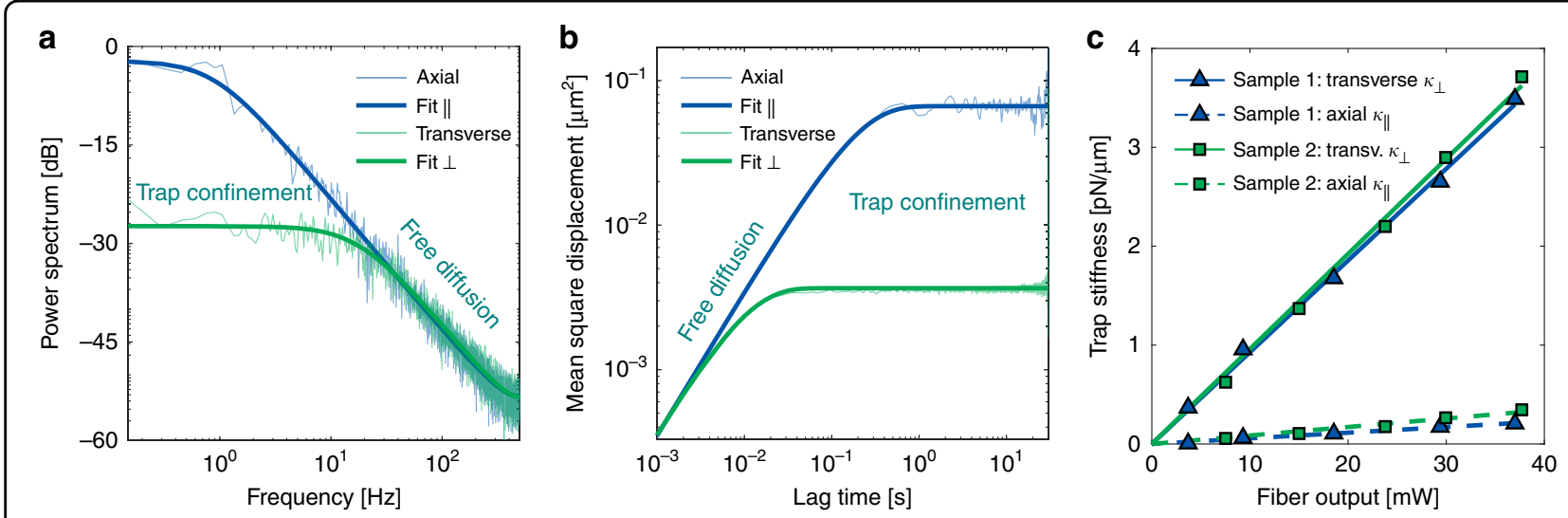

Fig. 5 Analysis of the results from trapping silica beads using the UNM-enhanced fibre. a Power-spectral-density (PSD) analysis of a representative dataset (shown in Fig. 4d), including the corresponding fits (thick lines) for the axial (blue) and transverse (green) directions. b Corresponding mean-square-displacement (MSD) analysis for the same dataset including fits. c Resulting trap stiffness at different power levels for both samples (sample 1: NA $=0.84$, sample 2: NA $=0.88$, for details, see Fig. 2b) determined using the MSD method (Fig. 5b). As expected, a higher NA yields stronger trapping, while the transverse stiffness is approximately one order of magnitude higher than that in the axial direction (compare ref. ${ }^{13}$ )

Overall, good agreement between MSD and PSD analysis for the retrieved fit parameters $\left(f_{\mathrm{c}}\right.$ and $D$ ) was achieved, and, thus, we solely discuss the MSD results in the following. The final trap stiffness for the two implemented samples (sample 1: $\mathrm{NA}=0.84$, sample 2: $\mathrm{NA}=$ 0.88 ) was determined at different power levels for the transverse and axial directions independently $\left(\kappa_{\perp}\right.$ and $\left.\kappa_{\|}\right)$ and is shown in Fig. 5c. The transverse stiffness $\kappa_{\perp}$ is approximately one order of magnitude larger than $\kappa_{||}$due to the elongated shape of the focus (ratio $>4: 1$ ), which directly impacts the distribution of the particle displacement (compare Figs. 3e and 4e). A comparison of the two implemented samples shows that sample 2 with $\mathrm{NA}=$ 0.88 exhibits a better trapping performance than sample 1 $(\mathrm{NA}=0.84)$, i.e., yields stronger trapping due to tighter focusing. Note that such quantitative analysis of the trajectory of the trapped $E$. coli was not conducted here owing to the additional presence of rotational diffusion, demanding a more complex tracking routine.

\section{Comparison to other work}

Considering previous work on meta-lens-enhanced fibres, it is worth mentioning that none of these approaches allows for optical trapping with a single fibre due to a low NA (Table 1). These methods demand the use of additional surfaces to overcome the axial scattering force $^{17,18,22}$, preventing their use for in vivo applications. Our UNM-enhanced fibre resolves this issue and demonstrates full 3D optical trapping with a single device. It exhibits an extraordinarily high numerical aperture, which we believe is the highest confirmed value for a meta-lens functionalized optical fibre to date allowing for optical trapping, as demonstrated here for dielectric microparticles and $E$. coli bacteria. Other approaches that principally reach trapping-relevant numerical apertures (Table 1) require sophisticated and noncommercially available fibres and extensive additional equipment (e.g., spatial light modulators), all of which are not required here. In contrast, the presented approach solely includes commercial SMFs and MMFs, yielding significant advantages such as low cost, fibre length independence, compatibility with fibre circuitry and robustness against external influences such as bending, all of which are limiting factors in the previous work ${ }^{14}$. Another key benefit is the straightforward implementation procedure based on nanoprinting and splicing, avoiding the employment of clean-room-type machinery, including mask exposure, multistep processing, and large-scale and cost-intensive equipment ${ }^{18,19,30-32}$. An additional remarkable feature is the high damage threshold of our device, which overall results from an extended beam cross-section at the metalens location, effectively reducing the local intensity to a maximum value of $I_{\text {peak }} \sim 6 \mathrm{~mW} / \mu \mathrm{m}^{2}$, which we found to be below the damage threshold. Note that stable trapping with each implemented sample was achieved over many trapping experiments (each of which lasted for minutes), and no sample degradation was observed over the course of weeks.

\section{Discussion}

In this work, we have demonstrated that combining a nanoprinted ultra-flat meta-lens with an end-face modified step-index SMF leads to the realization of a novel type of strongly focusing meta-fibre device exhibiting a record-high numerical aperture of up to 0.9 and diffraction-limited spot sizes, allowing the optical trapping 
Table 1 Summary of the previously reported work

\begin{tabular}{|c|c|c|c|c|c|c|c|}
\hline $\begin{array}{l}\text { Working } \\
\text { principle }\end{array}$ & Fabrication method & $\begin{array}{l}\text { Lens } \\
\text { material }\end{array}$ & Fibre type & Wavelength & Measured NA & $\begin{array}{l}\text { Trapping } \\
\text { application }\end{array}$ & Reference \\
\hline $\begin{array}{l}\text { Refractive } \\
\text { microprism }\end{array}$ & Two-photon lithography & Polymer & 4 SMF bundle & $1070 \mathrm{~nm}$ & $\begin{array}{l}1.15 \text { water } \\
\text { (theor.) }\end{array}$ & $\begin{array}{l}\text { Red blood/ } \\
\text { tumour cells }\end{array}$ & ref. ${ }^{51}$ \\
\hline $\begin{array}{l}\text { Diffractive } \\
\text { meta-lens }\end{array}$ & fs direct laser writing & Polymer & $\begin{array}{l}1 \mathrm{SMF}+\mathrm{MMF} \\
\text { spliced }\end{array}$ & $660 \mathrm{~nm}$ & $\begin{array}{l}0.882 \\
\text { water }\end{array}$ & $\begin{array}{l}2 \mu \mathrm{m} \text { beads/ } \\
\text { E. coli }\end{array}$ & This work \\
\hline $\begin{array}{l}\text { Refractive } \\
\text { ball lens }\end{array}$ & Glue & $\mathrm{SiO}_{2}$ & $\begin{array}{l}1 \mathrm{SMF}+\mathrm{MMF} \\
\text { spliced }\end{array}$ & $980 \mathrm{~nm}$ & $\begin{array}{l}0.875 \\
\text { water }\end{array}$ & $\begin{array}{l}0.2 \mu \mathrm{m} \text { beads/ } \\
\text { yeast cells }\end{array}$ & ref. ${ }^{52,53}$ \\
\hline Digital holography & Spatial light modulator & - & $1 \mathrm{MMF}$ & $1064 \mathrm{~nm}$ & $\begin{array}{l}>0.8 \\
\text { water }\end{array}$ & $1.5 \mu \mathrm{m}$ beads & ref. ${ }^{14}$ \\
\hline $\begin{array}{l}\text { Diffractive } \\
\text { meta-lens }\end{array}$ & $\begin{array}{l}\text { fs direct laser } \\
\text { writing }\end{array}$ & Polymer & $\begin{array}{l}2 \mathrm{SMFs}+\text { spacer } \\
\text { printed }\end{array}$ & $808 \mathrm{~nm}$ & $\begin{array}{l}0.7 \\
\text { water }\end{array}$ & $\begin{array}{l}1 \mu \mathrm{m} / \\
0.5 \mu \mathrm{m} \text { beads }\end{array}$ & ref. ${ }^{22}$ \\
\hline $\begin{array}{l}\text { Plasmonic } \\
\text { nanorods }\end{array}$ & Focused ion beam milling & $\mathrm{Au}$ & $1 \mathrm{PCF}$ & $1550 \mathrm{~nm}$ & $\begin{array}{l}0.37 \\
\text { air }\end{array}$ & - & ref. ${ }^{18}$ \\
\hline $\begin{array}{l}\text { Refractive } \\
\text { GRIN lens }\end{array}$ & $\begin{array}{l}\text { Stack \& draw } \\
+ \text { glue }\end{array}$ & $\mathrm{SiO}_{2}$ & $\begin{array}{l}1 \text { SMF + } \\
\text { spacer glued }\end{array}$ & $976 \mathrm{~nm}$ & $\begin{array}{l}0.16 \\
\text { air }\end{array}$ & $\begin{array}{l}2 \mu \mathrm{m} \text { beads } \\
\text { (on the surface) }\end{array}$ & ref. ${ }^{54}$ \\
\hline $\begin{array}{l}\text { Refractive } \\
\text { microlens }\end{array}$ & Laser exposure & Polymer & $\begin{array}{l}1 \mathrm{SMF}+\mathrm{MMF} \\
\text { spliced }\end{array}$ & $980 \mathrm{~nm}$ & $?$ & $\begin{array}{l}8 \mu \mathrm{m} \text { beads/ } \\
\text { yeast cells } \\
\text { (on the surface) }\end{array}$ & ref. ${ }^{55}$ \\
\hline $\begin{array}{l}\text { Diffractive } \\
\text { Fresnel plate }\end{array}$ & Focused ion beam milling & $\mathrm{SiO}_{2}$ & $\begin{array}{l}1 \mathrm{SMF}+\mathrm{MMF} \\
\text { spliced }\end{array}$ & $980 \mathrm{~nm}$ & $?$ & $\begin{array}{l}8 \mu \mathrm{m} \text { beads/ } \\
\text { yeast cells } \\
\text { (on the surface) }\end{array}$ & ref. ${ }^{17}$ \\
\hline $\begin{array}{l}\text { Diffractive } \\
\text { Fresnel plate }\end{array}$ & $\begin{array}{l}\text { UV-nanoimprint } \\
\text { lithography }\end{array}$ & Polymer & $1 \mathrm{SMF}$ & $660 \mathrm{~nm}$ & $?$ & - & ref. ${ }^{30}$ \\
\hline $\begin{array}{l}\text { Refractive } \\
\text { microaxicon }\end{array}$ & HF chemical etching & $\mathrm{SiO}_{2}$ & $1 \mathrm{SMF}$ & $633 \mathrm{~nm}$ & $?$ & - & ref. ${ }^{19}$ \\
\hline
\end{tabular}

Our work is highlighted in grey

of microbeads and bacteria with a single SMF in combination with diffractive optics for the first time. The exceptionally high numerical aperture of the meta-lens enhanced fibre and its capability to optically trap objects with a single meta-fibre device enables one to envision applications in areas that are currently inaccessible by state-of-the-art devices. In contrast to electron beam lithography, 3D nanoprinting allows for applications in a wide range of fields due to the fast and cost-effective implementation of biocompatible devices that can be straightforwardly adapted to specific requirements. Potential fields of application include fibre-optic-based confocal microscopy, beam collimation for waveguide coupling, fibre lasers, fibre-based trapping and manipulation in quantum technology, bioanalytics, and life sciences. With respect to the latter, the introduced meta-fibre device may represent a first feasible pathway towards in vivo applications that include strong light focusing operation in difficult-to-access areas, e.g., high-resolution scanning-based tissue imaging.
Further improvements of the UNM design in terms of NA can be achieved by using narrowband laser sources and by more precise cleaving of the MMF or better characterization of the extended beam, both of which reduce the overcompensation of the wavefront curvature (Fig. 3e, g). The spatial resolution of the DLW system used $(\Delta x=$ $300 \mathrm{~nm}$ ) limits the meta-lens phase profile discretization, which can be improved by recent advancements in DLW technology. As reported in ref. ${ }^{33}$, stimulated-emissiondepletion $^{34}$ yields resolutions down to $\Delta x=9 \mathrm{~nm}$, allowing for further enhancement of the NA, as suggested by Fig. $2 \mathrm{~b}$. Increasing the refractive index of the liquid medium represents another way to increase the NA (e.g., by using immersion oil ${ }^{35}$ ), while here, we chose water to maintain biocompatibility and applicability. Note that dimethyl sulfoxide is an interesting candidate that is widely used in biology, with a refractive index as high as that of fused silica $^{36}$. In addition, the concepts demonstrated in refs. ${ }^{37-39}$ provide a new perspective for further increasing the transmission efficiency and controlling the chromatic aberration 
of meta-fibres. Furthermore, we envision the use of our UNM as a fibre-optic endoscope, as demonstrated in ref. ${ }^{40}$, and consider the use of novel materials ${ }^{41}$.

\section{Materials and methods}

\section{Experimental design and sample preparation}

The fibre facet was functionalized by a silanization process prior to DLW, leading to better chemical and mechanical stability of the $\mathrm{UNM}^{42}$ and improved adhesion between the $\mathrm{SiO}_{2}$ and a polymeric microstructure ${ }^{43}$. To remove organic residue, the fibre tip was cleaned before by successively sonicating in acetone, isopropyl alcohol, and distilled water ( $15 \mathrm{~min}$ each) and drying with nitrogen. Subsequently, oxygen plasma activation was performed for $1 \mathrm{~min}(150 \mathrm{~W})$ followed by overnight immersion in 1\% 3-(trimethoxysilyl)propyl methacrylate (Sigma-Aldrich 440159) dissolved in ethanol and drying with nitrogen. The on-fibre UNM was fabricated by using a commercial DLW system with a $63 \times$ objective (NA = 1.4) to polymerize the negative photosensitive IP-Dip resist (Nanoscribe $\mathrm{GmbH}$ ). For high-resolution 3D nanoprinting, we chose the commercial IP-Dip photoresist that offers a high spatial resolution down to $300 \mathrm{~nm}$. Alternatively, IP-L photoresist with stronger mechanical stability but a reduced spatial resolution of $500 \mathrm{~nm}$ can also be used for creating 3D nanopillar-based birefringent metasurfaces $^{44}$, which can potentially extend the metafibre capabilities for controlling the additional degrees of freedom of light, including amplitude, polarization, and orbital angular momentum. A $5 \mu \mathrm{m}$-thick base layer was first printed to promote the strong mechanical connection of the lens and fibre surface, followed by the actual printing of the UNM $(50 \mathrm{~nm}$ hatching, $200 \mathrm{~nm}$ slicing, approximate printing time $1 \mathrm{~h}$ ). After printing, the onfibre UNM was developed in a propylene glycol monomethyl ether acetate (PGMEA, Sigma-Aldrich 484431) bath for $20 \mathrm{~min}$ followed by a 2 min Novec $^{\text {TM }}$ (SigmaAldrich SHH0002) rinse. The final sample was rinsed with isopropyl alcohol before measurement.

\section{Focal scan measurement}

The focus characterization relies on in-coupling a $\mathrm{CW}$ laser diode $\left(\lambda_{0}=660 \mathrm{~nm}, \Delta \lambda_{0}=13 \mathrm{~nm}, \quad\right.$ Thorlabs L660P120) to the water-immersed sample and imaging the beam from the front via a water-dipping objective (Nikon $60 \times, \mathrm{NA}=1.0$ ) and a subsequent achromatic tube lens $(f=300 \mathrm{~mm})$ onto a CMOS camera (Basler acA640$750 \mathrm{uc}$, pixel size $4.8 \mu \mathrm{m}$ ). The focal position of the objective is axially scanned with a piezo-driven objective collar (Mad City Labs Nano-F100) in steps of size $\Delta z=$ $100 \mathrm{~nm}$. At each position, micrographs are taken for three different exposure times $(0.1 \mathrm{~ms}, 1 \mathrm{~ms}$, and $10 \mathrm{~ms})$, which are combined afterwards in a postprocessing routine to create HDR images with a noise floor smaller than $10^{-5}$.

\section{Optical trapping procedure}

For the optical trapping experiments, light from a CW laser diode $\left(\lambda_{0}=660 \mathrm{~nm}\right.$ (Thorlabs L660P120), shown in Fig. 4a) is coupled into the meta-fibre device, leading to an output power of $P_{\text {out }}=37 \mathrm{~mW}$ after the fibre meta-lens combination. The functionalized fibre tip is placed inside a water-filled chamber containing the particle solution $(2 \mu \mathrm{m}$ silica beads, Micromod Inc., concentration $c \sim 5 \cdot 10^{6} \mathrm{~cm}^{-3}$ ) consisting of two parallel microscope cover glasses spaced $\sim 0.5 \mathrm{~mm}$ apart. The motion of a trapped bead is recorded from the side via a home-built microscope (50x Olympus dry objective in combination with an $f=200 \mathrm{~mm}$ tube lens, resulting in $55 \times$ magnification) using a high-speed camera (Basler piA640-210m, pixel size $7.4 \mu \mathrm{m})$. A fast frame rate $(f=1 \mathrm{kHz})$ and a very small exposure time $(\tau=10 \mu \mathrm{s})$ are used to minimize motion blur. Homogeneous particle illumination was provided by a Koehler-type arrangement (wavelength of $455 \mathrm{~nm}$, as shown in Fig. 4c) consisting of an LED coupled to a delivery MMF $(\mathrm{NA}=0.22$, the core diameter of $105 \mu \mathrm{m}$ (Thorlabs FG105LCA)). The light emitted from the MMF is first collimated using an $f=6.24 \mathrm{~mm}$ lens followed by a beam de magnifying telescope $(f=150 \mathrm{~mm}$ and $f=8 \mathrm{~mm}$ ), resulting in the final illumination with $\mathrm{NA}=0.16$ and a spot size of $150 \mu \mathrm{m}$.

\section{Trajectory determination}

For evaluation of the trapped particle behaviour, the trap stiffness is determined from recordings at different power levels by introducing neutral density (ND) filters before the in-coupling side of the fibre. As shown in Fig. $4 \mathrm{~d}$, the trajectory of a trapped bead is presented, extracted by centring each frame at time $t$ via a Fourier shift algorithm assuming mirror symmetry (see ref. ${ }^{13}$ for details). This yields the displacement $x_{\perp,||}(t)$ in the directions perpendicular $\left(x_{\perp}=y\right)$ and parallel $\left(x_{\|}=z\right)$ to the fibre axis and ultimately results in the complete track of a trapped particle on timescales of $>1$ min.

\section{Statistical analysis and details of the fitting routine}

The calculated PSD presented in Fig. 5a is first compressed by binning via Welch's method to reduce error from noise and correlation ${ }^{45-47}$. Afterwards, it is fitted to the power spectrum $\operatorname{PSD}(f)=2 D /\left(4 \pi^{2}\left(f_{\mathrm{c}}{ }^{2}+\right.\right.$ $\left.\left.f^{2}\right)\right)+\varepsilon^{2}$ via a maximum likelihood estimate for statistically correlated data ${ }^{46-48}$ to retrieve the fit parameters, namely, corner frequency $f_{\mathrm{c}}$ (where the power spectrum drops to half) and the free particle diffusion coefficient $D$. In comparison, Fig. 5b illustrates the corresponding MSD calculated for the same particle displacement $x_{\perp,||}(t)$ and fitted using $\operatorname{MSD}(\Delta t)=2 D /$ $\left(2 \pi f_{\mathrm{c}}\right) \cdot\left(1-\exp \left(-2 \pi f_{\mathrm{c}} \Delta t\right)\right)+\varepsilon^{2}$ including weights to compensate for correlation ${ }^{29,48-50}$. The error, $\varepsilon^{2}$, results from uncertainty in tracking the particle position and is a 
measure of the motion blur due to excessively long exposure times. Here, the exposure time was chosen to be very small $(\tau=10 \mu \mathrm{s})$.

\section{Acknowledgements}

M.P. and J.K. acknowledge funding from the Leibniz Science Campus InfectoOptics (Whole Blood Imaging project SAS-2015) and the DFG, projects SCHM2655/8-1 and SCHM2655/15-1. R.F. acknowledges financial support from the WW foundation. H.R. and S.A.M. acknowledge support from the Alexandervon-Humboldt Foundation and the Lee-Lucas Chair in Physics. M.P., R.F., and M.A.S. thank R. Heintzmann for important comments on the fibre design and J. Rüger, I. Leite and S. Turtaev for lending equipment and technical assistance with the focal scan measurement. The authors also thank R. Fatobene Ando for visual implementation of Fig. 1a and J. Dellith for help with SEM imaging.

\section{Author details}

${ }^{1}$ Leibniz Institute of Photonic Technology, 07745 Jena, Germany. ${ }^{2}$ Abbe Center of Photonics and Faculty of Physics, FSU Jena, 07745 Jena, Germany. ${ }^{3}$ Chair in Hybrid Nanosystems, Nanoinstitute Munich, LMU München, 80539 München, Germany. ${ }^{4}$ Department of Physics, Imperial College London, London SW7 2AZ, UK. ${ }^{5}$ Otto Schott Institute of Material Research, FSU Jena, 07745 Jena, Germany

\section{Author contributions}

M.P. performed the experiments and analysed the results. M.P., J.K., and H.R. designed and manufactured the samples. R.F. contributed to the

understanding of the design and showing its limits. M.A.S. and S.A.M. led the project and proposed the research idea. M.P. and M.A.S. wrote the manuscript with contributions from all authors.

\section{Funding}

Open Access funding enabled and organized by Projekt DEAL.

\section{Data availability}

The data that support the plots within this article and other findings of this study are available from the corresponding author upon reasonable request.

\section{Conflict of interest}

The authors declare no conflict of interest.

Supplementary information The online version contains supplementary material available at https://doi.org/10.1038/s41377-021-00491-z.

Received: 8 September 2020 Revised: 8 December 2020 Accepted: 10 February 2021

Published online: 15 March 2021

\section{References}

1. Maragò, O. M. et al. Optical trapping and manipulation of nanostructures. Nat. Nanotechnol. 8, 807-819 (2013).

2. Ashkin, A. \& Dziedzic, J. M. Optical trapping and manipulation of viruses and bacteria. Science 235, 1517-1520 (1987).

3. Wang, M. D. et al. Stretching DNA with optical tweezers. Biophys. J. 72 1335-1346 (1997)

4. Guck, J. et al. The optical stretcher: a novel laser tool to micromanipulate cells. Biophys. J. 81, 767-784 (2001).

5. Johansen, P. L. et al. Optical micromanipulation of nanoparticles and cells inside living zebrafish. Nat. Commun. 7, 10974 (2016).

6. Korda, P. T. et al. Kinetically locked-in colloidal transport in an array of optical tweezers. Phys. Rev. Lett. 89, 128301 (2002).

7. MacDonald, M. P., Spalding, G. C. \& Dholakia, K. Microfluidic sorting in an optical lattice. Nature 426, 421-424 (2003).

8. Ladavac, K., Kasza, K. \& Grier, D. G. Sorting mesoscopic objects with periodic potential landscapes: optical fractionation. Phys. Rev. E 70, 010901 (2004)

9. Beugnon, J. et al. Two-dimensional transport and transfer of a single atomic qubit in optical tweezers. Nat. Phys. 3, 696-699 (2007).
10. Reimann, R. et al. GHz rotation of an optically trapped nanoparticle in vacuum. Phys. Rev. Lett. 121, 033602 (2018).

11. Constable, A. et al. Demonstration of a fiber-optical light-force trap. Opt. Lett. 18, 1867-1869 (1993).

12. Solmaz, M. E. et al. Optical stretching of giant unilamellar vesicles with an integrated dual-beam optical trap. Biomed. Opt. Express 3, 2419-2427 (2012).

13. Plidschun, M. et al. Nanobore fiber focus trap with enhanced tuning capabilities. Opt. Express 27, 36221-36230 (2019).

14. Leite, I. T. et al. Three-dimensional holographic optical manipulation through a high-numerical-aperture soft-glass multimode fibre. Nat. Photon. 12, 33-39 (2018).

15. Khorasaninejad, M. et al. Metalenses at visible wavelengths: diffraction-limited focusing and subwavelength resolution imaging. Science 352, 1190-1194 (2016).

16. Zeisberger, M. et al. Nanoboomerang-based inverse metasurfaces-a promising path towards ultrathin photonic devices for transmission operation. APL Photon. 2, 036102 (2017).

17. Ribeiro, R. S. R. et al. Fabrication of Fresnel plates on optical fibres by FIB milling for optical trapping, manipulation and detection of single cells. Sci. Rep. 7, 4485 (2017).

18. Yang, J. Y. et al. Photonic crystal fiber metalens. Nanophotonics 8, 443-449 (2019).

19. Kuchmizhak, A. et al. High-quality fiber microaxicons fabricated by a modified chemical etching method for laser focusing and generation of Bessel-like beams. Appl. Opt. 53, 937-943 (2014).

20. Gissibl, T. et al. Sub-micrometre accurate free-form optics by threedimensional printing on single-mode fibres. Nat. Commun. 7, 11763 (2016).

21. Gissibl, T., Schmid, M. \& Giessen, H. Spatial beam intensity shaping using phase masks on single-mode optical fibers fabricated by femtosecond direct laser writing. Optica 3, 448-451 (2016).

22. Asadollahbaik, $\mathrm{A}$. et al. Highly efficient dual-fiber optical trapping with 3D printed diffractive Fresnel lenses. ACS Photon. 7, 88-97 (2020).

23. Neuman, K. C. \& Block, S. M. Optical trapping. Rev. Sci. Instrum. 75, 2787-2809 (2004).

24. Yamada, $\mathrm{K}$. et al. Multilevel phase-type diffractive lenses in silica glass induced by filamentation of femtosecond laser pulses. Opt. Lett. 29, 1846-1848 (2004).

25. Siemion, A. Terahertz diffractive optics-smart control over radiation. J. Infrared, Millim. 40, 477-499 (2019).

26. Shannon, C. E. Communication in the presence of noise. Proc. IRE 37, 10-21 (1949).

27. Amos, B., McConnell, G. \& Wilson, T. Confocal microscopy. in Comprehensive Biophysics (ed Egelman, E.) (Amsterdam: Elsevier, 2012).

28. Kramers, H. A. Brownian motion in a field of force and the diffusion model of chemical reactions. Physica 7, 284-304 (1940).

29. Förster, R. et al. Tracking and analyzing the Brownian motion of Nano-objects inside hollow core fibers. ACS. Sensors 5, 879-886 (2020).

30. Koshelev, A. et al. High refractive index Fresnel lens on a fiber fabricated by nanoimprint lithography for immersion applications. Opt. Lett. 41, 3423-3426 (2016).

31. Wang, N. et al. Nanotrimer enhanced optical fiber tips implemented by electron beam lithography. Optical Mater. Express 8, 2246-2255 (2018).

32. Wang, $\mathrm{N}$. et al. Boosting light collection efficiency of optical fibers using metallic nanostructures. ACS Photonics 6, 691-698 (2019).

33. Gan, Z. S. et al. Three-dimensional deep sub-diffraction optical beam lithography with 9 nm feature size. Nat. Commun. 4, 2061 (2013).

34. Fischer, J. \& Wegener, M. Three-dimensional direct laser writing inspired by stimulated-emission-depletion microscopy [Invited]. Optical Mater. Express 1, 614-624 (2011).

35. Liang, H. W. et al. Ultrahigh numerical aperture metalens at visible wavelengths. Nano Lett. 18, 4460-4466 (2018).

36. Kozma, I. Z., Krok, P. \& Riedle, E. Direct measurement of the group-velocity mismatch and derivation of the refractive-index dispersion for a variety of solvents in the ultraviolet. J. Optical Soc. Am. B 22, 1479-1485 (2005).

37. Chen, L. W., Li, Y. \& Hong, M. H. Total reflection metasurface with pure modulated signal. Adv. Opt. Mater. 7, 1801130 (2019).

38. Li, X. et al. Multicolor 3D meta-holography by broadband plasmonic modulation. Sci. Adv. 2, e1601102 (2016).

39. Dou, K. H. et al. Off-axis multi-wavelength dispersion controlling metalens for multi-color imaging. Opto-Electron. Adv. 3, 190005 (2020). 
40. Pahlevaninezhad, $\mathrm{H}$. et al. Nano-optic endoscope for high-resolution optical coherence tomography in vivo. Nat. Photon. 12, 540-547 (2018).

41. Lin, $\mathrm{H}$. et al. Diffraction-limited imaging with monolayer $2 \mathrm{D}$ material-based ultrathin flat lenses. Light:: Sci. Appl. 9, 137 (2020).

42. Schade, M. et al. Chemical functionalization of carbon/polymer bipolar plate materials via oxygen plasma activation and subsequent silanization. Surf. Coat. Technol. 240, 255-260 (2014)

43. Liu, X. J. et al. 3D printing of bioinspired liquid superrepellent structures. $A d v$. Mater. 30, 1800103 (2018).

44. Ren, H. R. et al. Complex-amplitude metasurface-based orbital angular momentum holography in momentum space. Nat. Nanotechnol. 15, 948-955 (2020).

45. Welch, $P$. The use of fast Fourier transform for the estimation of power spectra: a method based on time averaging over short, modified periodograms. IEEE Trans. Audio Electroacoust. 15, 70-73 (1967).

46. Berg-Sørensen, K. \& Flyvbjerg, H. Power spectrum analysis for optical tweezers. Rev. Sci. Instrum. 75, 594-612 (2004).

47. Lansdorp, B. M. \& Saleh, O. A. Power spectrum and Allan variance methods for calibrating single-molecule video-tracking instruments. Rev. Sci. Instrum. 83, 025115 (2012)
48. Nørrelykke, S. F. \& Flyvbjerg, H. Power spectrum analysis with least-squares fitting: amplitude bias and its elimination, with application to optical tweezers and atomic force microscope cantilevers. Rev. Sci. Instrum. 81, 075103 (2010).

49. Saxton, M. J. \& Jacobson, K. Single-particle tracking: applications to membrane dynamics. Annu. Rev. Biophys. Biomol. Struct. 26, 373-399 (1997).

50. Ruthardt, N., Lamb, D. C. \& Bräuchle, C. Single-particle tracking as a quantitative microscopy-based approach to unravel cell entry mechanisms of viruses and pharmaceutical nanoparticles. Mol. Ther. 19, 1199-1211 (2011).

51. Liberale, C. et al. Integrated microfluidic device for single-cell trapping and spectroscopy. Sci. Rep. 3, 1258 (2013).

52. Tang, X. Y. et al. Super-low-power optical trapping of a single nanoparticle. Opt. Lett. 44, 5165-5168 (2019).

53. Zhang, Y. et al. 3-dimensional dark traps for low refractive index bio-cells using a single optical fiber Bessel beam. Opt. Lett. 43, 2784-2786 (2018).

54. Kasztelanic, R. et al. Integrating free-form nanostructured GRIN Microlenses with single-mode fibers for optofluidic systems. Sci. Rep. 8, 5072 (2018).

55. Rodrigues, S. M. et al. Fabrication of multimode-single mode polymer fiber tweezers for single cell trapping and identification with improved performance. Sensors 18, 2746 (2018). 